\title{
A Modified Floor Field Model and Pareto Optimum of Pedestrian Evacuation Efficiency
}

\author{
Yan Xu, ${ }^{1}$ Li-Jun Tian, ${ }^{2}$ and Gui Yong ${ }^{1}$ \\ ${ }^{1}$ School of Statistics and Mathematics, Inner Mongolia University of Finance and Economics, Hohhot 010070, China \\ ${ }^{2}$ School of Economics and Management, Fuzhou University, Fuzhou 350108, China \\ Correspondence should be addressed to Gui Yong; nmyonggui@163.com
}

Received 3 December 2016; Accepted 30 May 2017; Published 27 June 2017

Academic Editor: Alessandro Gasparetto

Copyright (c) 2017 Yan Xu et al. This is an open access article distributed under the Creative Commons Attribution License, which permits unrestricted use, distribution, and reproduction in any medium, provided the original work is properly cited.

\begin{abstract}
A modified floor field model is proposed to simulate the pedestrian evacuation behavior in a room with multiple exits. The modification relies upon introduction of a so-called semidynamical floor field which additionally embodies two cognition coefficients related to exit width and pedestrian distribution around exits. The energy consumption and time requirement of evacuees are numerically investigated and the Pareto optimum of evacuation efficiency is obtained by selecting the combinations of the two cognition coefficients.
\end{abstract}

\section{Introduction}

Study on pedestrian dynamics has attracted many physicists over the past few years. Various collective behaviors and self-organization phenomena have been observed from the view point of complexity of the pedestrian flow system. For understanding these phenomena, many models have been developed, for example, the social force model [1-3], the fluid dynamic model [4-9], and the cellular automata model [1021].

The social force model is a deterministic continuum model in which the interactions between pedestrians are governed by the social force or social field [1,2]. Using this model, such phenomena as the arching around exit, the lane formation in counter flow, and the oscillation of flow direction at narrow door can be reproduced.

The fluid dynamic model describes how density and velocity change over time with the use of partial differential equation. This model can be used to investigate exactly the dynamics at the exits during an evacuation [7] and study some typical pedestrian behaviors, for example, the "thinking fluid" behavior and the overcompression effect $[8,9]$.

The rule-based cellular automata model is discrete in space and time. All pedestrians are represented by particles. Such typical phenomena as arching, jamming, kin behavior, faster-is-slower, lane formation, and sidle effect can be simulated by this kind of models [10-21]. Floor field model is one kind of discrete simulation models. At each time step, every pedestrian moves from one lattice to the neighboring lattice in terms of a transition probability. This model can be used to investigate various collective effects and the self-organization encountered in pedestrian dynamics $[10,11]$.

Modeling the evacuation process of pedestrians in a room with multiple exits has to consider the strategy of selecting exits. In the conventional floor field models, the exit selection strategy is mainly based on the herding behavior and the use of knowledge about the shortest path to each exit $[10,11]$. In some works, the occupant density around each exit is considered as an important factor affecting the exit selection strategy $[15,16]$. In this paper, we improve the floor field model through introducing two cognition coefficients associated with the exit width and the pedestrian distributions around exits, respectively.

The energy consumed in evacuation process is seldom studied by previous researches. However, if a space for evacuation is large enough to make some pedestrians expend more energy than their capabilities, he or she will fail to evacuate successfully and, on the contrary, they may become obstacles to other pedestrians. In this study, we will investigate the energy consumption of evacuees besides the 
time requirement and find the Pareto optimum of evacuation efficiency.

\section{Model}

Let a space for evacuation be represented by two-dimensional square lattices. The size of each lattice is approximately $40 \times$ $40 \mathrm{~cm}^{2}$. Each lattice can be either empty or occupied by one pedestrian. Each time step is real time of evacuation based on different movement velocity of pedestrians. The length of one time step is 0.3 seconds in this study. This implies a walking speed of approximately $1.33 \mathrm{~m} / \mathrm{s}$.

In each time step, pedestrians move only one lattice site in the forward, backward, left, or right direction or remain unmoving. They probabilistically select a neighboring lattice site in these directions to move using the transition probability. The transition probability is computed using the static and dynamic floor fields of those lattices.

In the simulation of evacuation process, the static floor field $S$ represents the degree of attractiveness of each lattice for pedestrians. The static floor field does not evolve with time and is not changed by the presence of the pedestrians. It is often given by the value depending on the distance from the exit. The dynamic floor field $D$ represents the characteristic that pedestrians tend to follow their predecessors. This is implemented using bosons dropped by pedestrians, having their own dynamics through diffusion and decay controlled by probabilities $\sigma$ and $\delta$. The dynamic floor field $D$ is the number of bosons remaining on the cell at each time step. These two types of floor fields have been historically used as fundamental and essential components of the floor field model.

The static floor field defined above reflects the attractiveness degree of each lattice for pedestrians, but the exit width and the pedestrian distribution around exits have not been considered. These two factors should have impacts on the attractiveness of some lattices and eventually influence the movement behavior of pedestrians. For this reason, the floor field model has to be improved so as to simulate the evacuation process more precisely.

2.1. Occupant Density. The occupant density around exit is introduced to characterize the pedestrian distribution around exits in the evacuation process. The occupant density $O_{r}^{m}$ of exit $m$ can be defined as the number of occupied cells in the effect area of exit $m$ with radius $r$. The effect area is a special region around the exit. Figure 1 provides an illustration for calculating the occupant density $O_{r}^{m}[16]$. The width of the exit is 2 cells. The effect area contains sixteen intact cells which are located within a half-round with radius of 4 cells. Five black colored cells are occupied. Thus, the occupant density of this exit is 5 .

2.2. Semidynamical Floor Field. The semidynamical floor field is introduced for considering the effects of exit width and pedestrian distribution around exit. Let $L$ be the total width of all exits, $l_{m}$ the width of exit $m$, and $T$ the total number of exits. We have $\sum_{m=1}^{T} l_{m}=L$.

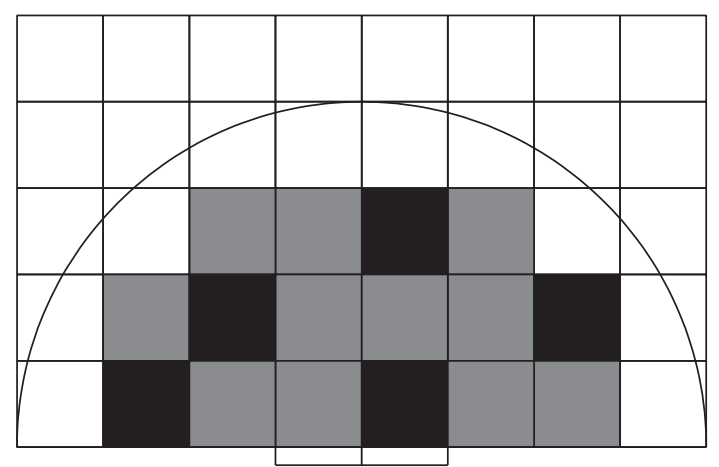

FIGURE 1: Illustration for calculating occupant density $O_{r}^{m}$.

The semidynamical floor field $S D_{i j}^{m}$ of lattice site $(i, j)$ to exit $m$ is defined as

$$
S D_{i j}^{m}=\max _{m} \max _{(k, h)}\left\{Z_{k h}^{m}\right\}-Z_{i j}^{m},
$$

where $(h, k)$ is a lattice. $Z_{i j}^{m}$ is determined by the shortest distance $M_{i j}^{m}$ from lattice $(i, j)$ to exit $m$, the width $l_{m}$ of exit $m$, and the occupant density $O_{r}^{m}$ of exit $m$, that is,

$$
Z_{i j}^{m}=M_{i j}^{m}+\alpha\left(L-l_{m}\right)+\beta O_{r}^{m}
$$

where $M_{i j}^{m}=\min _{n} \sqrt{\left(i-i_{n}^{m}\right)^{2}+\left(j-j_{n}^{m}\right)^{2}},\left(i_{n}^{m}, j_{n}^{m}\right)$ is the coordinate of the $n$th cell of exit $m$. The parameter $\alpha$ is a cognition coefficient which indicates the pedestrian's capability of perceiving the effect of exit width. A larger $\alpha$ value means a bigger consideration of the effect of exit width or pedestrian being more sensible to the width effect. The parameter $\beta$ is another cognition coefficient which indicates the pedestrian's sensitivity to the people distribution around each exit. A larger $\beta$ means more consideration on the occupant density. When $\alpha=0$ and $\beta=0$, the effects of both exit width and pedestrian distribution are not considered and the semidynamical floor field is reduced to the static floor field.

2.3. Transition Probability. The transition probability from a lattice site to its neighboring lattice site $(i, j)$ toward exit $m$, $P_{i j}^{m}$, is computed using the following:

$$
P_{i j}^{m}=N \exp \left(k_{S D} S D_{i j}^{m}\right) \exp \left(k_{D} D_{i j}^{m}\right)\left(1-\mu_{i j}\right) \xi_{i j},
$$

where $N$ is a normalization factor for ensuring $\sum_{(i, j)} P_{i j}^{m}=1$. $k_{S D}$ and $k_{D}$ are two sensitivity parameters for scaling $S D_{i j}^{m}$ and $D_{i j}^{m}$, respectively. The value of $k_{S D}$ can be regarded as a measure of the pedestrians' perception about the attractiveness of each lattice site for each exit. The parameter $k_{D}$ for coupling to the dynamic field governs the tendency to follow the lead of others. A large value of $k_{D}$ implies a strong herding behavior. The parameter $\mu_{i j}$ indicates whether the neighboring lattice $(i, j)$ is occupied or not. It equals 1 if the lattice is occupied 
and 0 otherwise. The parameter $\xi_{i j}$ is related to the existence of obstacles. It is 0 if $(i, j)$ is occupied by obstacles (e.g., walls) and 1 otherwise.

2.4. Unit Energy Consumed. We use the total movement steps of all pedestrians, rather than the total evacuation time, to represent the energy consumption. This says that when a pedestrian moves to one of neighboring lattices, one unit of energy consumption is added to the counter. The average energy consumed per person is termed as unit energy consumed. These two performance indexes are generally not equivalent to each other because, in congested situation, some pedestrians may not move if their neighboring sites are occupied by others while the time is spent. In addition, if some pedestrians change the exit choice frequently in the evacuation process, the energy consumption may increase although the evacuation time is reduced.

2.5. Pareto Optimum. The multicriteria optimization method has widely been applied in designing various mechanical structures and dynamic systems. Due to the basic nature of conflict among multiple objectives, a set of compromising solutions called Pareto optima exists. The determination of this set is essential for decision-makers to understand the trade-off relationship among alternatives [21].

In this study, we use two criteria, namely, the unit energy consumed and the evacuation time, to measure the efficiency of evacuation mechanism. We investigate how the two criteria are affected by the cognition coefficients $\alpha$ and $\beta$. We certainly hope to achieve such a result that these two indexes are simultaneously minimized by optimizing the two parameters $\alpha$ and $\beta$. However, this is basically impossible as discussed in the previous subsection, and we have to turn to finding the Pareto optimum of the evacuation mechanism.

\section{Simulation}

Consider a special zone in a room and suppose pedestrians are randomly distributed in the zone and attempt to escape. Without loss of generality, in Figure 2, the room is discretized into $50 \times 50$ cells and has four exits allocated at the centre of each wall. The width of exit $n$ is $n$ cells, $n=1,2,3,4$. In the specified zone (the small square marked by dotted line, with a size $22 \times 22$ cells), there are 400 pedestrians at the initial stage of the simulation.

The sensitivity parameters adopted here are $k_{S D}=3$ and $k_{D}=0.5$. The decay probability is $\sigma=0.5$ and the diffusion probability $\delta=0.5$. The radius of effect area is $r=10$ cells. We carry out 10 simulations for each set of parameters and compute the averages.

To investigate the role of each exit, the pedestrian number evacuated from each exit is shown in Figures 3 and 4, when $\alpha$ and $\beta$ take different values. It can be observed from Figures $3(\mathrm{a})-3(\mathrm{~d})$ that, for each $\alpha$ value, with increase of $\beta$ value, Exits 2 and 3 are first chosen by many pedestrians and then by less ones. This is because the special zone is located closer to these two exits and pedestrians do not care much about the occupant densities around exits. A larger $\beta$ value means

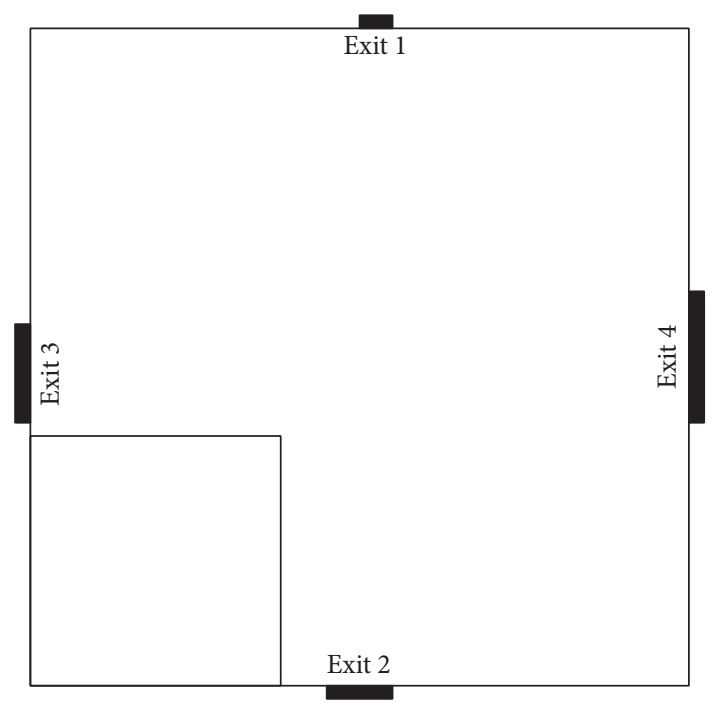

Figure 2: Room structure.

pedestrians are more sensitive to the occupant densities; as a result, some pedestrians turn to choosing exits 1 and 4 . Another important finding is that the $\alpha$ value influences the number of pedestrians evacuated from each exit, but the influence degree is not linear and monotonic.

Figure 4 shows that, with the increase of $\alpha$ value, for each $\beta$ value, the numbers of pedestrians evacuated from Exits 1 and 2 decrease, the one from Exit 4 increases, and the one from Exit 3 first ascends and then descends basically. This is because in the evacuation process, the occupant density in the effect area of each exit intends to be identical and the cognitive coefficient $\alpha$ gradually comes to play a role. Consequently, more pedestrians turn to evacuating from Exit 4 due to its width.

Figure 5 shows that the unit energy consumed monotonically increases with $\beta$ for each $\alpha$ value. This verifies such a view point that the energy consumption is proportional to the attitude toward congestion degree.

Finally, we investigate the two criteria for evaluating the evacuation efficiency. Due to the conflict of the two criteria, it is difficult to find the optimal $\alpha$ and $\beta$ values that simultaneously minimize the unit energy consumed and evacuation time. For solving this problem, the Pareto concept is used here. Figure 6 depicts these two indexes against combination of $\alpha$ and $\beta$ values. The Pareto optimum points are $\left(\alpha^{*}, \beta^{*}\right) \in\{(0,0.4),(0,0.3),(1,0.3),(3,0.3)$, $(4,0.3),(5,0.3),(0,0.2),(1,0.2),(1,0),(0,0.1),(1,0.1)\}$.

\section{Conclusions}

In this paper, two criteria, namely, the unit energy consumed and the evacuation time, are exploited to evaluate the evacuation mechanism. The unit energy consumed is seldom mentioned by previous researchers. An improved floor field model is proposed to simulate the evacuation process in a room with multiple exits where pedestrians are distributed 

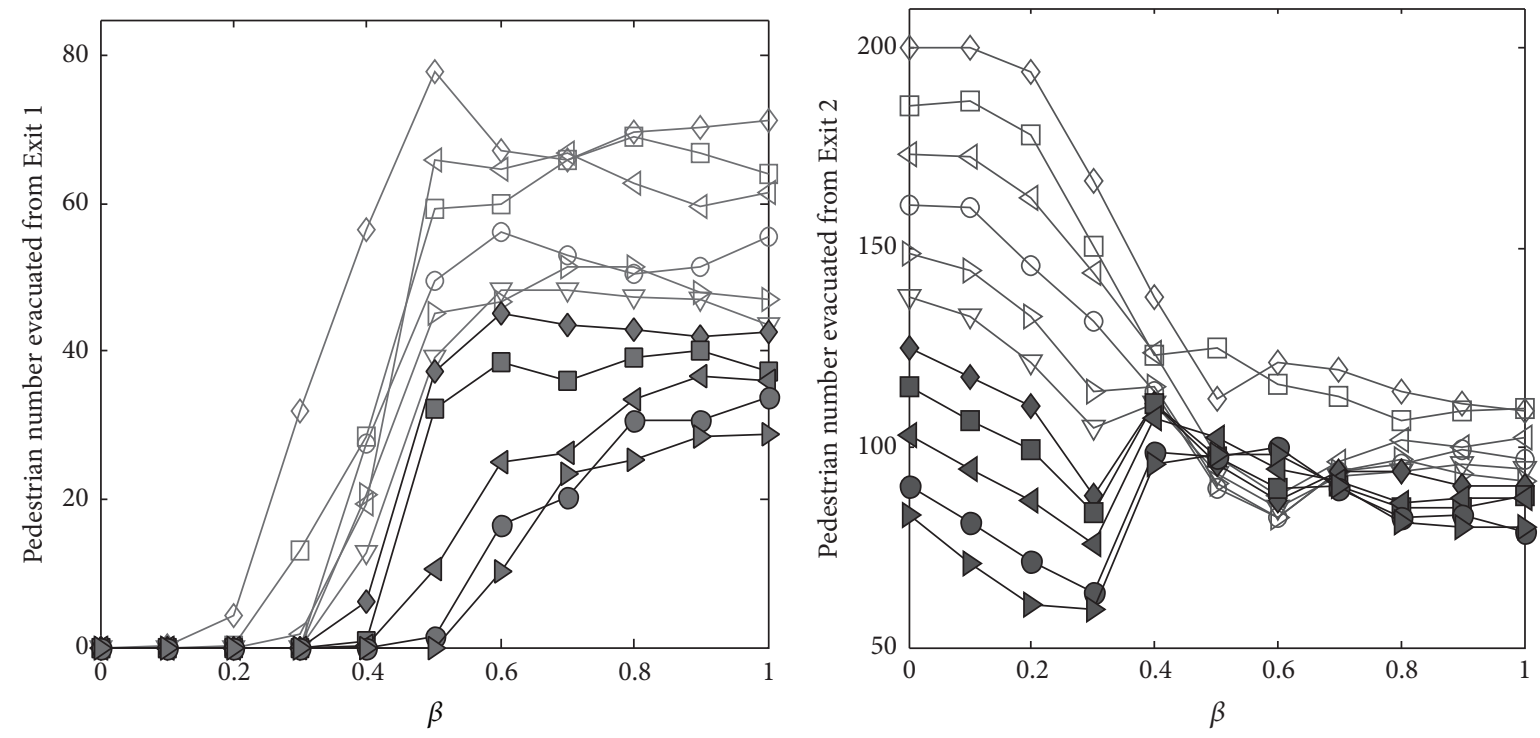

$$
\begin{aligned}
& \neg \alpha=0 \\
& \square \alpha=1 \\
& \neg \alpha=2 \\
& \neg \alpha=3 \\
& \neg \alpha=4 \\
& \nabla \alpha=5
\end{aligned}
$$

(a)

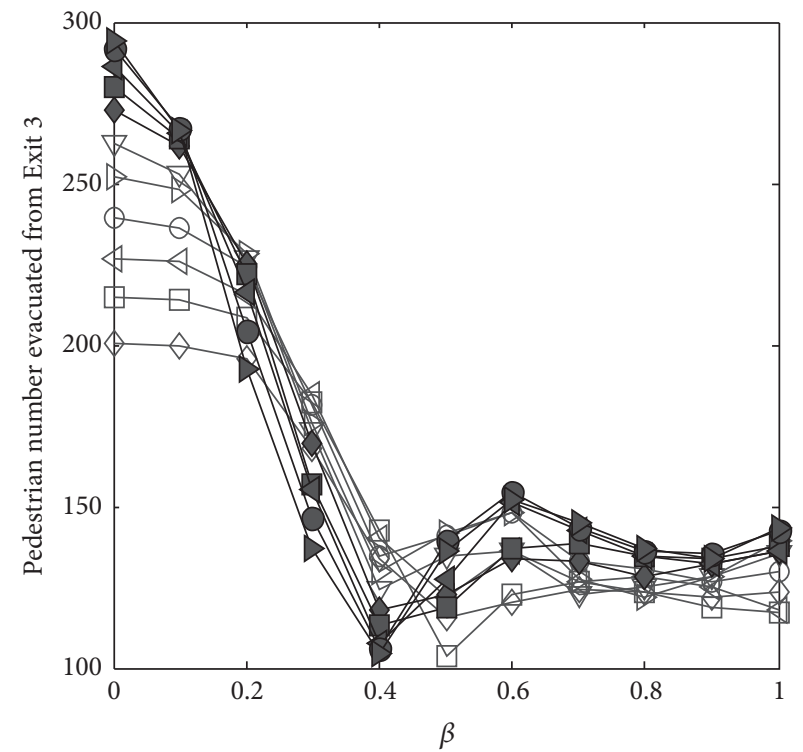

$\diamond \alpha=0$

$\square \alpha=1$

$\dashv \alpha=2$

$-\alpha=3$

$\rightarrow \alpha=4$

$\nabla \alpha=5$

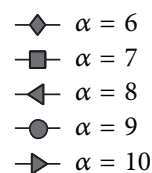

$$
\begin{aligned}
& \neg \alpha=0 \\
& \square \alpha=1 \\
& \neg \alpha=2 \\
& \neg \alpha=3 \\
& \neg \alpha=4 \\
& \nabla \alpha=5
\end{aligned}
$$

(b)

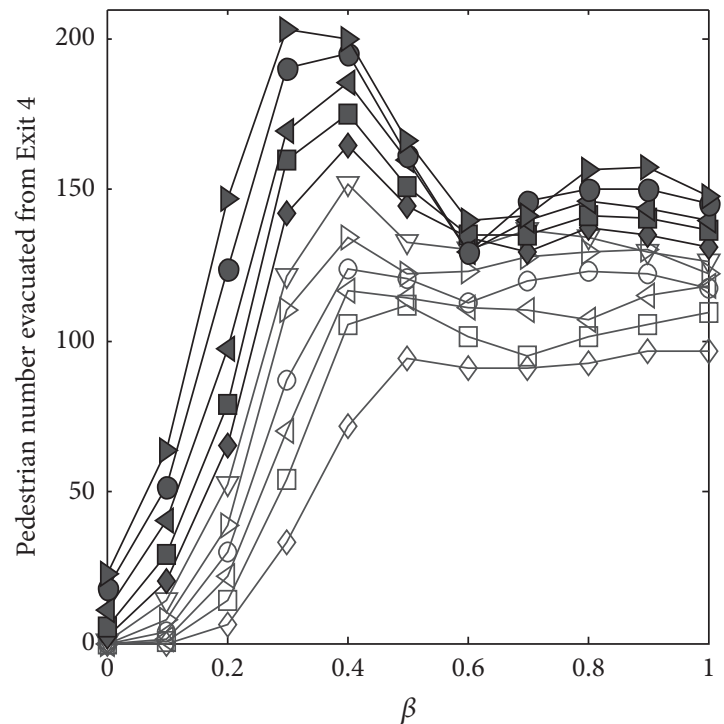

$$
\begin{aligned}
& \neg \alpha=0 \\
& \square \alpha=1 \\
& \neg \alpha=2 \\
& \neg \alpha=3 \\
& \neg \alpha=4 \\
& \nabla \alpha=5
\end{aligned}
$$

(c)

Figure 3: Pedestrian number evacuated from each exit against cognition coefficient $\beta$ for different $\alpha$ values. 


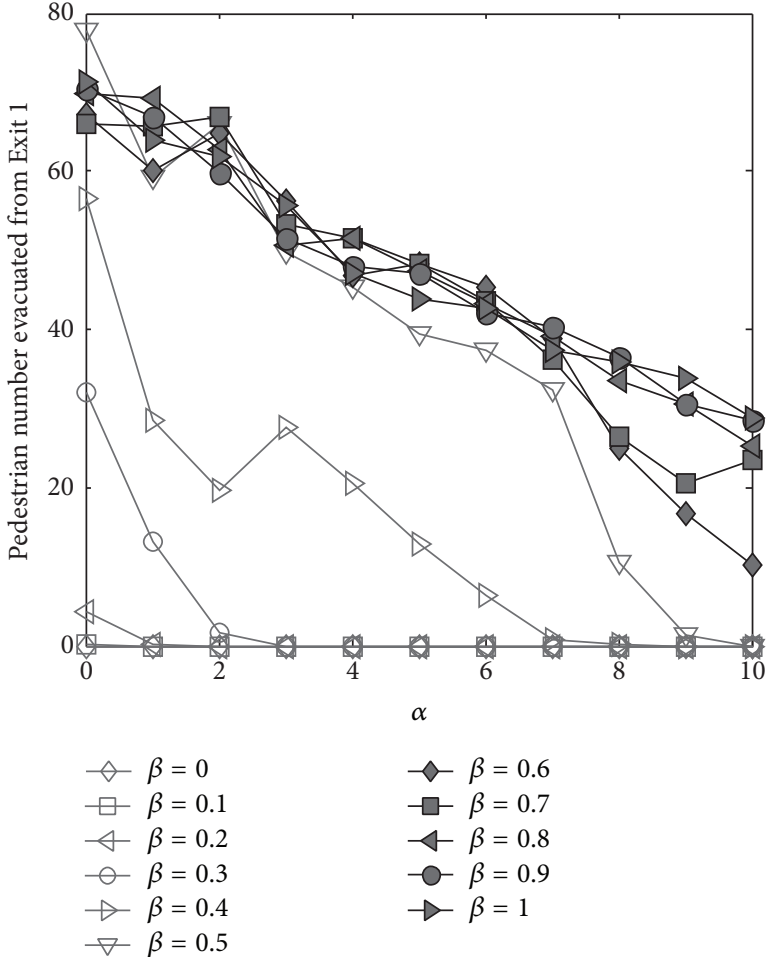

(a)

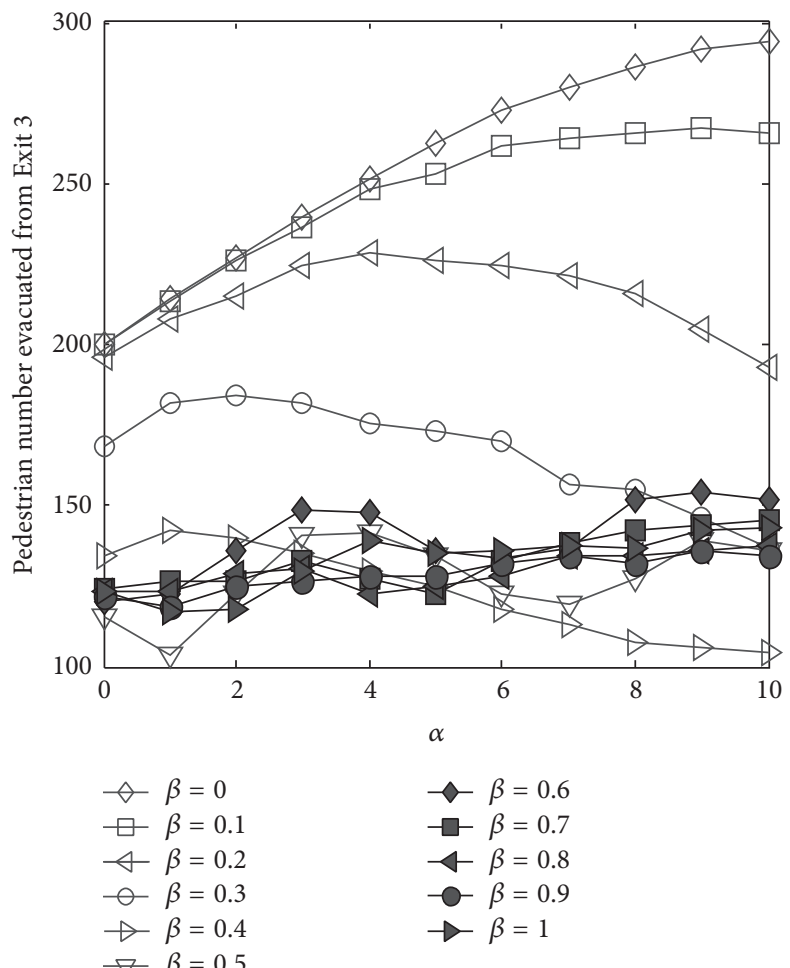

(c)

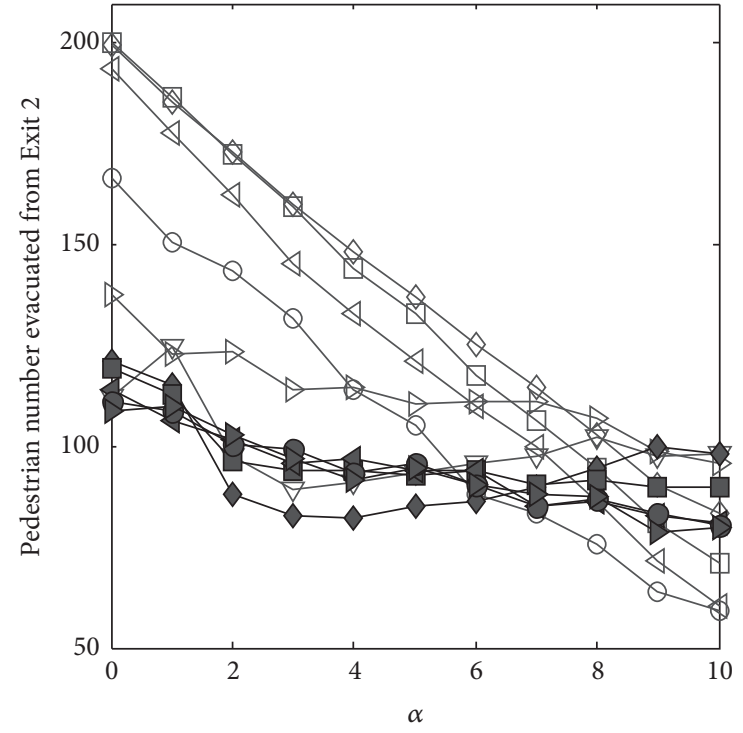

$\neg \beta=0$
$\square \beta=0.1$
$\neg \beta=0.2$
$\neg \beta=0.3$
$\neg \beta=0.4$
$\nabla \beta=0.5$

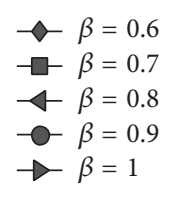

(b)

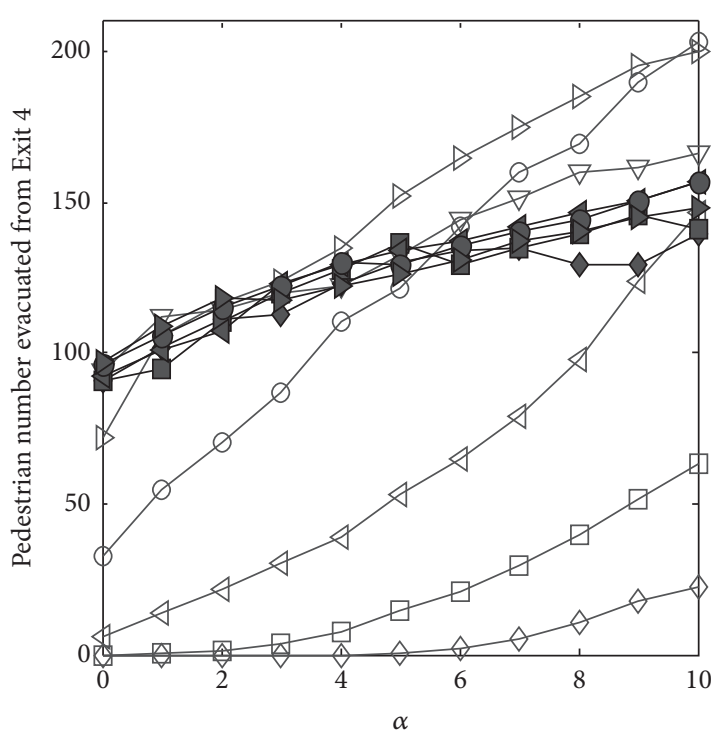

$$
\begin{aligned}
& \neg \beta=0 \\
& \square \beta=0.1 \\
& \neg \beta=0.2 \\
& \bigcirc \beta=0.3 \\
& \neg \beta=0.4 \\
& \nabla \beta=0.5
\end{aligned}
$$$$
\begin{aligned}
& \neg-\beta=0.6 \\
& \neg-\beta=0.7 \\
& \neg \beta=0.8 \\
& \multimap \beta=0.9 \\
& \neg \beta=1
\end{aligned}
$$

(d)

Figure 4: Pedestrian number evacuated from each exit against cognition coefficient $\alpha$ for different $\beta$ values. 


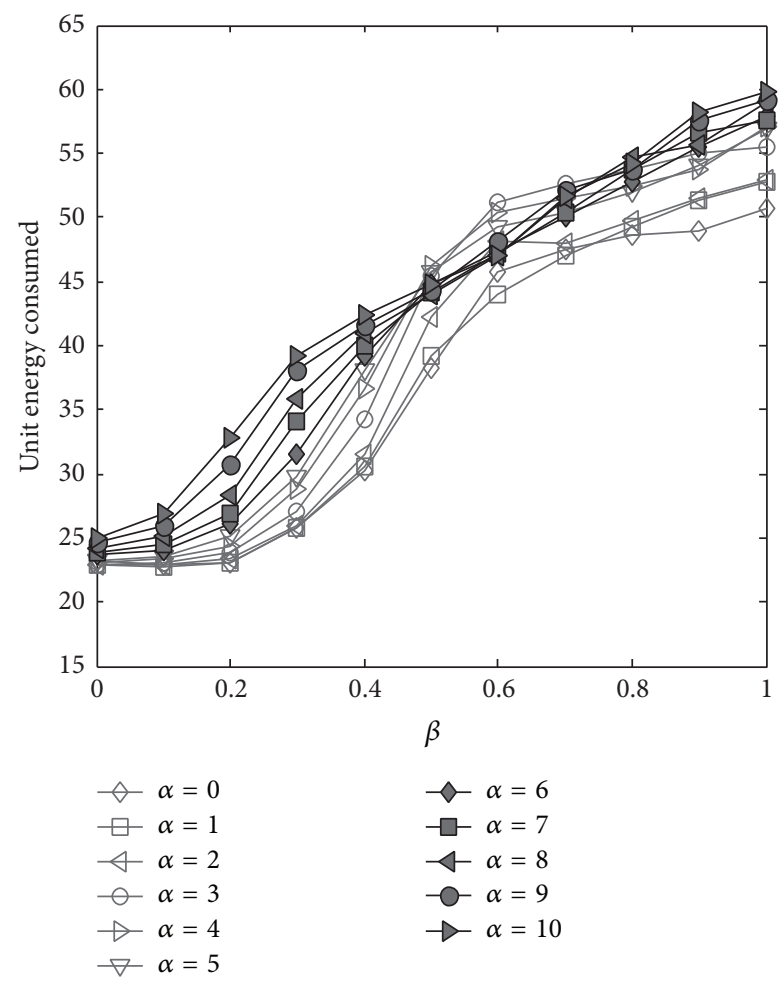

FIGURE 5: Unit energy consumed against $\beta$ for different $\alpha$ values.

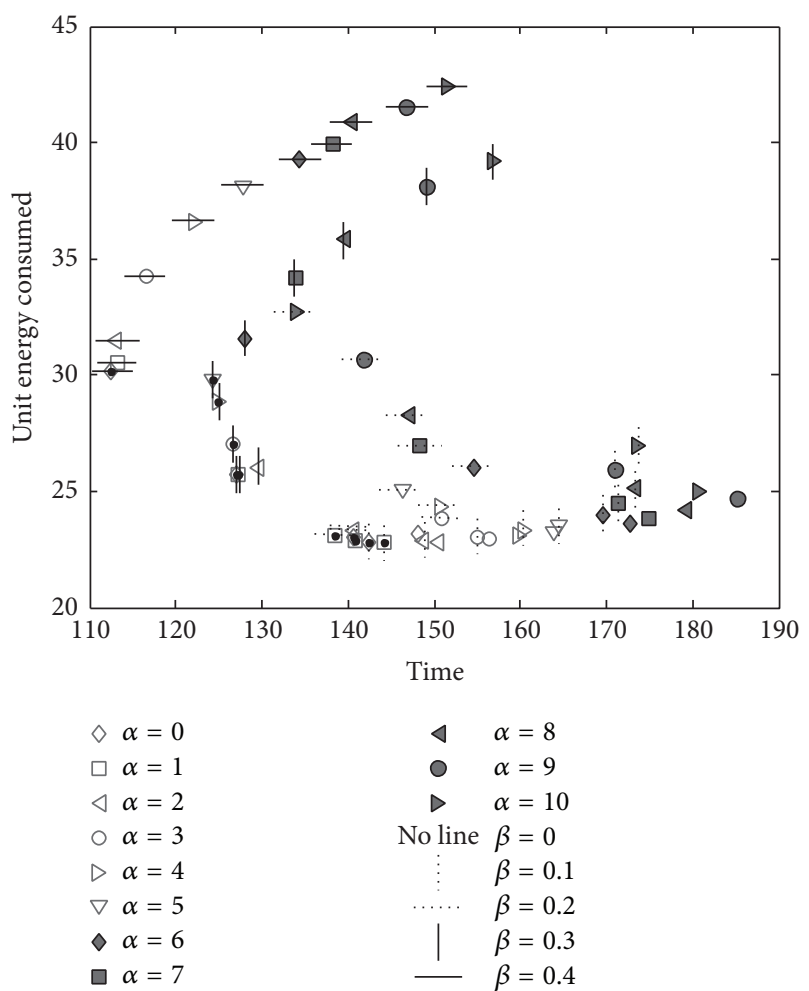

Figure 6: Pareto optima between unit energy consumed and evacuation time. heterogeneously. The improvement relies on the modification to the conventional static floor field by incorporating such new factors as exit width and the pedestrian distribution around exits. Simulation results show that the evacuation capacity of each exit is sensitive to the pedestrians' cognition to these two factors. The Pareto optima are numerically obtained, which verifies the conflict of the two criteria.

\section{Conflicts of Interest}

The authors declare that there are no conflicts of interest regarding the publication of this paper.

\section{Acknowledgments}

This study was supported by the National Natural Science Foundation of China (Grants nos. 71401083, 71301028, and 71661024) and the Program for Young Talents of Science and Technology in Universities of Inner Mongolia Autonomous Region (NJYT15B06).

\section{References}

[1] D. Helbing and P. Molnar, "Social force model for pedestrian dynamics," Physical Review E, vol. 51, no. 5, pp. 4282-4286, 1995.

[2] D. Helbing, I. Farkas, and T. Vicsek, "Simulating dynamical features of escape panic," Nature, vol. 407, no. 6803, pp. 487490,2000

[3] W. Zeng, P. Chen, H. Nakamura, and M. Iryo-Asano, "Application of social force model to pedestrian behavior analysis at signalized crosswalk," Transportation Research Part C: Emerging Technologies, vol. 40, pp. 143-159, 2014.

[4] L. F. Henderson, "The statistics of crowd fluids," Nature, vol. 229, no. 5284, pp. 381-383, 1971.

[5] Y.-Q. Jiang, W. Zhang, and S.-G. Zhou, "Comparison study of the reactive and predictive dynamic models for pedestrian flow," Physica A: Statistical Mechanics and its Applications, vol. 441, Article ID 16364, pp. 51-61, 2016.

[6] X. Zheng, T. Zhong, and M. Liu, "Modeling crowd evacuation of a building based on seven methodological approaches," Building and Environment, vol. 44, no. 3, pp. 437-445, 2009.

[7] B. Andreianov, C. Donadello, and M. D. Rosini, "Crowd dynamics and conservation laws with nonlocal constraints and capacity drop," Mathematical Models and Methods in Applied Sciences, vol. 24, no. 13, pp. 2685-2722, 2014.

[8] R. L. Hughes, "A continuum theory for the flow of pedestrians," Transportation Research Part B: Methodological, vol. 36, no. 6, pp. 507-535, 2002.

[9] R. M. Colombo and M. D. Rosini, "Pedestrian flows and nonclassical shocks," Mathematical Methods in the Applied Sciences, vol. 28, no. 13, pp. 1553-1567, 2005.

[10] C. Burstedde, K. Klauck, A. Schadschneider, and J. Zittartz, "Simulation of pedestrian dynamics using a two-dimensional cellular automaton," Physica A: Statistical Mechanics and Its Applications, vol. 295, no. 3-4, pp. 507-525, 2001.

[11] A. Kirchner and A. Schadschneider, "Simulation of evacuation processes using a bionics-inspired cellular automaton model for pedestrian dynamics," Physica A: Statistical Mechanics and its Applications, vol. 312, no. 1-2, pp. 260-276, 2002. 
[12] Y. F. Yu and W. G. Song, "Cellular automaton simulation of pedestrian counter flow considering the surrounding environment," Physical Review E: Statistical, Nonlinear, and Soft Matter Physics, vol. 75, no. 4, Article ID 046112, 2007.

[13] H. J. Huang and R. Y. Guo, "Static floor field and exit choice for pedestrian evacuation in rooms with internal obstacles and multiple exits," Physical Review E: Statistical, Nonlinear, and Soft Matter Physics, vol. 78, no. 2, Article ID 021131, 6 pages, 2008.

[14] X. Yang, B. Wang, and Z. Qin, "Floor Field Model Based on Cellular Automata for Simulating Indoor Pedestrian Evacuation," Mathematical Problems in Engineering, vol. 2015, Article ID 820306, 10 pages, 2015.

[15] Y. Xu and H.-J. Huang, "Simulation of exit choosing in pedestrian evacuation with consideration of the direction visual field," Physica A: Statistical Mechanics and its Applications, vol. 391, no. 4, pp. 991-1000, 2012.

[16] H. Zhao and Z. Gao, "Reserve capacity and exit choosing in pedestrian evacuation dynamics," Journal of Physics. A. Mathematical and Theoretical, vol. 43, no. 10, Article ID 105001, 10 pages, 2010.

[17] R.-Y. Guo and H.-J. Huang, "Logit-based exit choice model of evacuation in rooms with internal obstacles and multiple exits," Chinese Physics B, vol. 19, no. 3, Article ID 030501, 2010.

[18] X. X. Jian and X. Zhang, "Potential field cellular automata model for pedestrian evacuation in a domain with a ramp," Mathematical Problems in Engineering, vol. 2014, Article ID 714267, 7 pages, 2014.

[19] R.-Y. Guo, H.-J. Huang, and S. C. Wong, "A potential field approach to the modeling of route choice in pedestrian evacuation," Journal of Statistical Mechanics: Theory and Experiment, vol. 2013, no. 2, Article ID P02010, 2013.

[20] G. H. Goldsztein, "A Mathematical Model of the Formation of Lanes in Crowds of Pedestrians Moving in Opposite Directions," Discrete Dynamics in Nature and Society, vol. 2015, Article ID 872807, 7 pages, 2015.

[21] W. H. Zhang and T. Gao, "A min-max method with adaptive weightings for uniformly spaced Pareto optimum points," Computers and Structures, vol. 84, no. 28, pp. 1760-1769, 2006. 


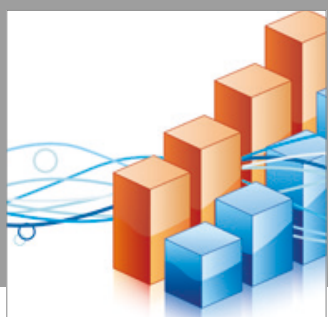

Advances in

Operations Research

vatersals

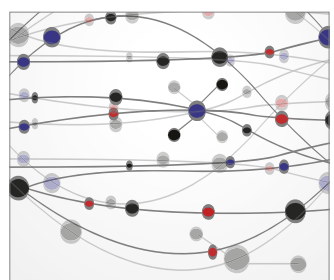

\section{The Scientific} World Journal
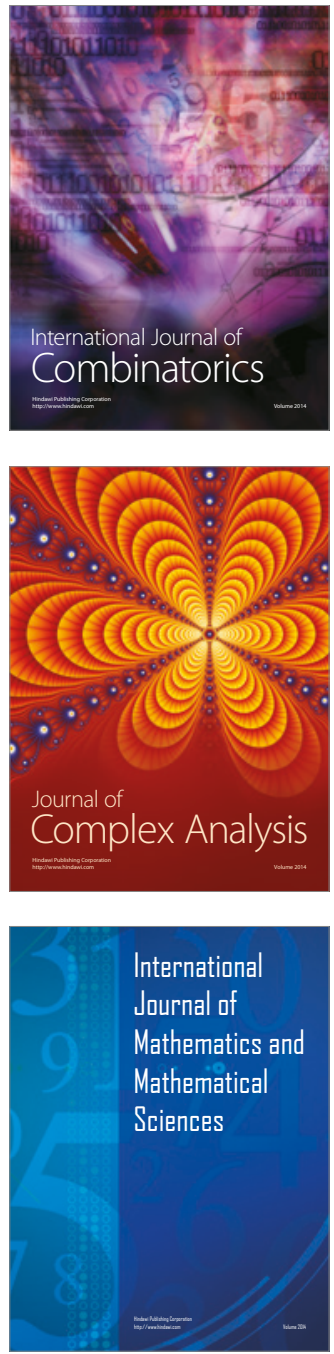
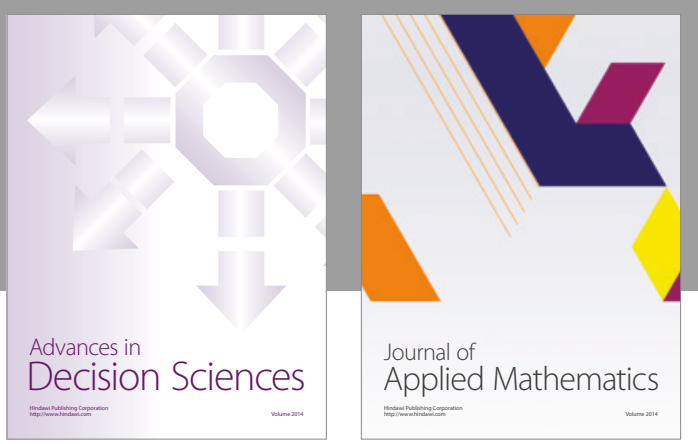

Algebra

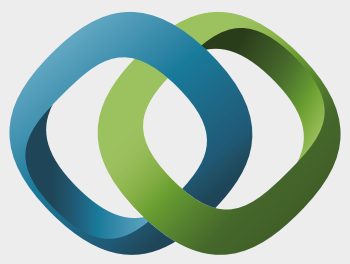

\section{Hindawi}

Submit your manuscripts at

https://www.hindawi.com
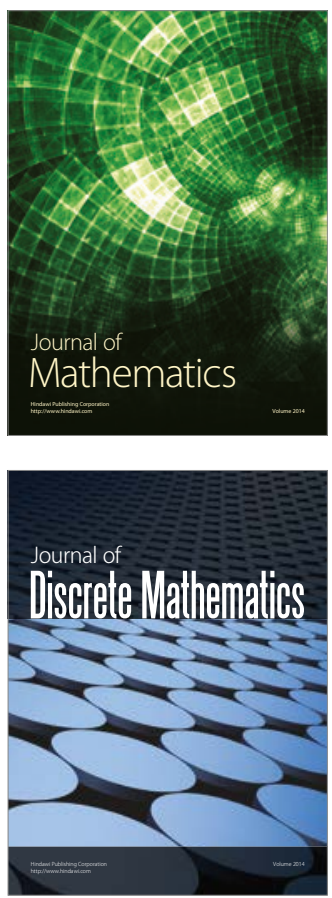

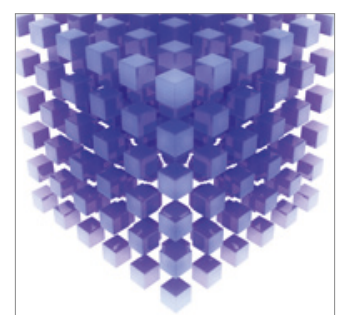

Mathematical Problems in Engineering
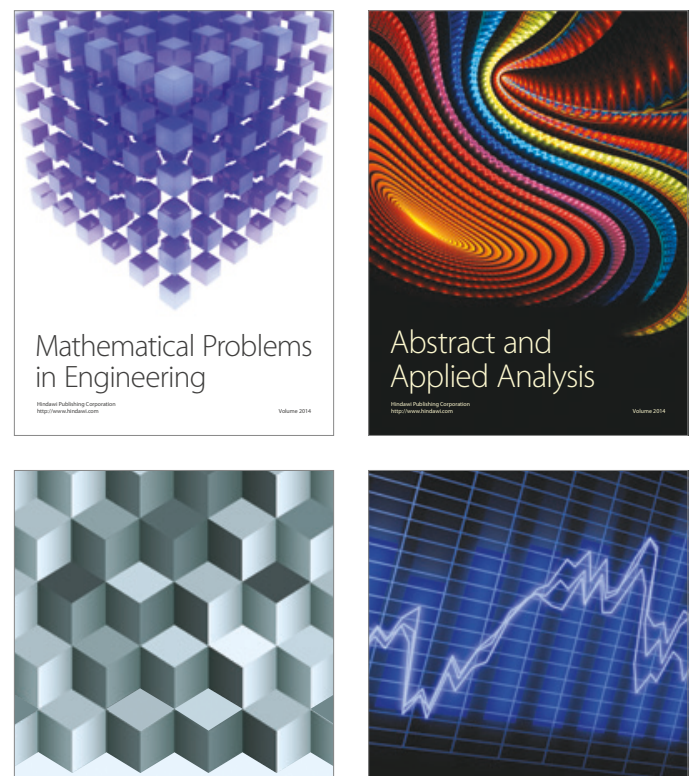

Journal of

Function Spaces

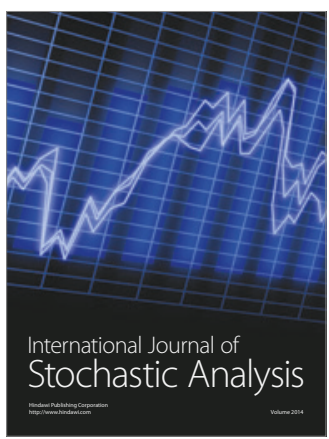

Probability and Statistics
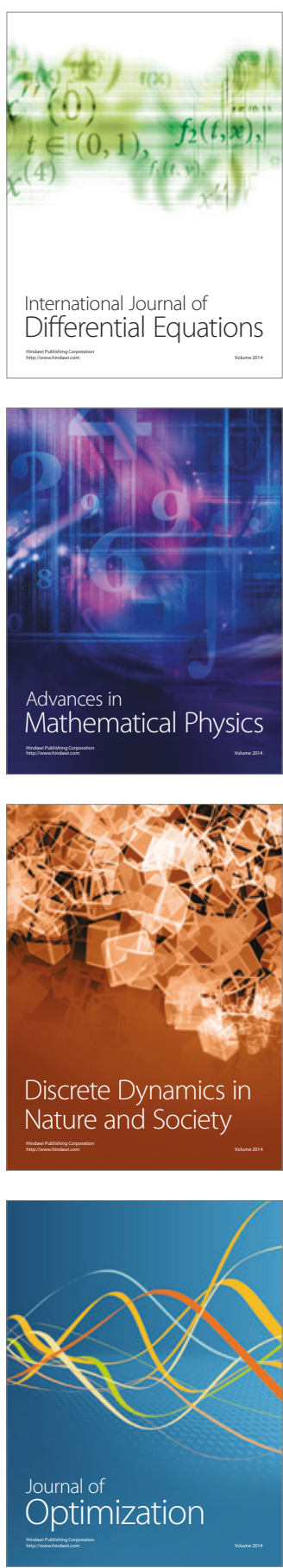